\title{
Asymptotic Completeness for a New Class of Stark Effect Hamiltonians
}

\author{
Arne Jensen* \\ Department of Mathematics, University of Kentucky, Lexington, KY 40506-0027, USA, and \\ Matematisk Institut, Aarhus Universitet, DK-8000 Aarhus C, Denmark
}

\begin{abstract}
Existence and completeness of the wave operators is shown for the Stark effect Hamiltonian in one dimension with a potential $V=W^{\prime \prime}$, where $W$ is a bounded function with four bounded derivatives. This class of potentials include some almost periodic functions and periodic functions with average zero over a period (Stark-Wannier Hamiltonians). In the last section we discuss classical particle scattering for the same class of potentials.
\end{abstract}

\section{Introduction. Statement of Result}

In this paper unitary equivalence of a class of one-dimensional Stark effect Hamiltonians with bounded potentials is shown. The new results are that no decay of the potential in the direction of the field is required and that the wave operators exist and are complete.

Let $H_{0}=-d^{2} / d x^{2}+x$ denote the free Stark effect Hamiltonian in $L^{2}(\mathbb{R})$. Let $B^{4}(\mathbb{R})$ denote the four times differentiable functions on $\mathbb{R}$ which are bounded with all derivatives bounded. The main result of this paper is

Theorem 1.1. Let $V=W^{\prime \prime}, W \in B^{4}(\mathbb{R})$, a real-valued function, and let $H=H_{0}+V$. Then the wave operators

$$
W_{ \pm}=\lim _{t \rightarrow \pm \infty} e^{i t H} e^{-i t H_{0}}
$$

exist and are unitary.

The wave operators give a unitary equivalence between $H$ and $H_{0} . H_{0}$ has purely absolutely continuous spectrum equal to $\mathbb{R}$, hence the same holds for $H$. Absence of singular continuous spectrum has been shown for a larger class of potentials in $[3,4,11]$. Absence of eigenvalues is a classical result from the theory of ordinary differential equations, see e.g. [5].

\footnotetext{
^ Partially supported by NSF-grant DMS-8401748
} 
There is a large literature on the existence and completeness of the wave operators for Stark effect Hamiltonians, see e.g. $[2,6,10,12,14]$. These authors all require a decay $V(x)=O\left(|x|^{-1 / 2-\varepsilon}\right)$ as $x \rightarrow-\infty$, i.e. in the direction of the electric field, to show existence and completeness of the wave operators. The class of potentials considered here include some almost period functions and sums of periodic functions with average zero over a period (Stark-Wannier Hamiltonians), see Sect. three. For recent results on the resonances in case $V$ is a trigonometric polynomial, see [1].

The proof is based on Mourre's version of the Enss method [8], and uses as a conjugate operator $A=i d / d x$, see [9]. The central part of our proof is to show that the class of $V$ considered here is short range with respect to the conjugate operator $A$. This result is obtained by a commutator method.

\section{Proofs}

Let $\mathscr{S}(\mathbb{R})$ denote the Schwartz space of rapidly decaying functions. In configuration space $L^{2}(\mathbb{R})$

$$
H_{0}=-\frac{d^{2}}{d x^{2}}+x
$$

is essentially self-adjoint on $\mathscr{S}(\mathbb{R})$. The operator

$$
A=i \frac{d}{d x}=-p
$$

is essentially self-adjoint on $\mathscr{S}(\mathbb{R})$ and is conjugate to $H_{0}$ at any point $E \in \mathbb{R}$ according to Mourre's definition [9], since we have

$$
i\left[H_{0}, A\right]=I
$$

on $\mathscr{S}(\mathbb{R})$. The propagation properties of $H_{0}$ with respect to $A$ can then be obtained from [7]. But since $H_{0}$ and $A$ satisfy the canonical commutation relation (2.3), the propagation estimates follow by an elementary computation. To state the results, let $P_{A}^{+}\left(P_{A}^{-}\right)$denote the spectral projections for $A$ corresponding to $(0, \infty)((-\infty, 0))$.

Proposition 2.1. For $s \geqq 0$ the following estimates hold:

$$
\begin{aligned}
& \left\|\left(A^{2}+1\right)^{-s / 2} e^{-i t H_{0}}\left(A^{2}+1\right)^{-s / 2}\right\| \leqq c(1+|t|)^{-s}, \quad t \in \mathbb{R}, \\
& \left\|\left(A^{2}+1\right)^{-s / 2} e^{-i t H_{0}} P_{A}^{ \pm}\right\| \leqq c(1+|t|)^{-s}, \quad \pm t>0 .
\end{aligned}
$$

Proof. We note that $e^{-i t H_{0}}$ maps $\mathscr{S}(\mathbb{R})$ into $\mathscr{S}(\mathbb{R})$. Using (2.3) we find

$$
e^{i t H_{0}} A e^{-i t H_{0}}=A+t,
$$

from which the results easily follow.

Mourre's version of the Enss method is based on the estimates (2.4) and (2.5). The result we need here is a special case and can be stated as follows. The proof can be found in $[8,10]$. See also [7], where an abstract short range scattering theory using a conjugate operator was given.

Proposition 2.2. Let $V$ be multiplication by a bounded real-valued function. Let 
$H=H_{0}+V$. Assume that $V$ satisfies

(A.1) $(H+i)^{-1}-\left(H_{0}+i\right)^{-1}$ is compact.

(A.2) There exist an integer $k \geqq 0$ and a real number $\delta>1$, such that the operator

$$
(H+i)^{-k} V\left(H_{0}+i\right)^{-k}\left(A^{2}+1\right)^{\delta / 2}
$$

extends to a bounded operator on $L^{2}(\mathbb{R})$. Then the wave operators

$$
W_{ \pm}=s-\lim e_{t \rightarrow \pm \infty}^{i t H} e^{-i t H_{0}}
$$

exist and are complete, i.e. the ranges of $W_{ \pm}$are equal to the subspace of absolute continuity of $H$. Furthermore, the singular continuous spectrum of $H$ is empty, and the point spectrum of $H$ is discrete in $\mathbb{R}$.

The proof of Theorem 1.1 is thus reduced to verifying (A.1) and (A.2). The key is the following lemma, whose proof is an elementary commutator computation. We let $B^{k}(\mathbb{R})$ denote the $k$-times continuously differentiable functions with all $k$ derivatives bounded on $\mathbb{R}$.

Lemma 2.3. Let $V$ be a real-valued function such that $V=W^{\prime \prime}, W \in B^{4}(\mathbb{R})$. Then we have:

(i) The operator $(H+i)^{-1} V p\left(H_{0}+i\right)^{-1}$ extends to a bounded operator on $L^{2}(\mathbb{R})$.

(ii) The operator $(H+i)^{-2} V p^{2}\left(H_{0}+i\right)^{-2}$ extends to a bounded operator on $L^{2}(\mathbb{R})$.

Proof. Let $U \in B^{2}(\mathbb{R})$. As a quadratic form on $\mathscr{S}(\mathbb{R}) \times \mathscr{S}(\mathbb{R})$ we have

$$
i\left[H_{0}, U\right]=2 U^{\prime} p-i U^{\prime \prime},
$$

and thus in the quadratic form sense on $\mathscr{S}(\mathbb{R}) \times \mathscr{S}(\mathbb{R})$

$$
\begin{aligned}
\frac{d}{d s} e^{i s H} U e^{-i s H_{0}} & =i e^{i s H}\left(H U-U H_{0}\right) e^{-i s H_{0}} \\
& =e^{i s H}\left(i V U+i\left[H_{0}, U\right]\right) e^{-i s H_{0}} \\
& =e^{i s H}\left(i V U+2 U^{\prime} p-i U^{\prime \prime}\right) e^{-i s H_{0}} .
\end{aligned}
$$

From this relation we get

$$
\begin{aligned}
& 2(H+i)^{-1} e^{i s H} U^{\prime} p e^{-i s H_{0}}\left(H_{0}+i\right)^{-1} \\
& \quad=(H+i)^{-1} e^{i s H}\left\{i H U-i U H_{0}-i V U+i U^{\prime \prime}\right\} e^{-i s H_{0}}\left(H_{0}+i\right)^{-1} .
\end{aligned}
$$

Since the right-hand side is a bounded operator on $L^{2}(\mathbb{R})$, the left-hand side extends to a bounded operator on $L^{2}(\mathbb{R})$. To get the first part of the lemma, we set $s=0$ and take $U=W^{\prime}$ such that $U^{\prime}=W^{\prime \prime}=V$.

For the second part we compute the second derivative in (2.6), again as a quadratic form on $\mathscr{S}(\mathbb{R}) \times \mathscr{S}(\mathbb{R})$, this time for $U=W$.

$$
\begin{aligned}
\frac{d^{2}}{d s^{2}} e^{i s H} W e^{-i s H_{0}}= & \frac{d}{d s} e^{i s H}\left(i V W+2 W^{\prime} p-i W^{\prime \prime}\right) e^{-i s H_{0}} \\
= & e^{i s H}\left\{i V\left(i V W+2 W^{\prime} p-i W^{\prime \prime}\right)\right. \\
& \left.+i\left[H_{0}, i V W+2 W^{\prime} p-i W^{\prime \prime}\right]\right\} e^{-i s H_{0}} \\
= & e^{i s H}\left\{4 W^{\prime \prime} p^{2}-4 i W^{(3)} p+2 i V W^{\prime} p+2 i(V W)^{\prime} p\right. \\
& \left.+(V W)^{\prime \prime}-V^{2} W+V W^{\prime \prime}-W^{(4)}-2 W^{\prime}\right\} e^{-i s H_{0}}
\end{aligned}
$$


To repeat the above argument we need to know that the coefficients to $p$ above are of the form $U^{\prime}$, as required in (2.6). By assumption $V=W^{\prime \prime}$. If we note

$$
2 V W^{\prime}=2 W^{\prime \prime} W^{\prime}=\left(\left(W^{\prime}\right)^{2}\right)^{\prime},
$$

we see that all coefficients to $p$ are of the required form, and we can repeat our argument to get part (ii).

Lemma 2.4. Assume $V=W^{\prime \prime}, W \in B^{4}(\mathbb{R})$. Then the operator $(H+i)^{-1} V\left(H_{0}+i\right)^{-1}$ is compact.

Proof. We note (see [2]) that the unitary operator $U=e^{i p^{3} / 3}$ has the property

$$
U H_{0} U^{-1}=x
$$

where $x$ is the multiplication operator on $L^{2}(\mathbb{R})$. Using this result, Lemma $2.3(\mathrm{i})$, and complex interpolation, we find that the operator

$$
(H+i)^{-1} V\left(H_{0}^{2}+1\right)^{-s / 2}\left(p^{2}+1\right)^{s / 2}
$$

is bounded on $L^{2}(\mathbb{R})$ for $0 \leqq s \leqq 1$. Now write

$$
\begin{aligned}
(H+i)^{-1} V\left(H_{0}+i\right)^{-1}= & \left((H+i)^{-1} V\left(H_{0}^{2}+1\right)^{-1 / 4}\left(p^{2}+1\right)^{1 / 4}\right) \\
& \cdot\left(\left(p^{2}+1\right)^{-1 / 4}\left(H_{0}^{2}+1\right)^{-1 / 4}\right) \\
& \cdot\left(\left(H_{0}^{2}+1\right)^{1 / 2}\left(H_{0}+i\right)^{-1}\right) .
\end{aligned}
$$

The first and last term are both bounded operators. The middle term is unitarily equivalent to $\left(p^{2}+1\right)^{-1 / 4}\left(x^{2}+1\right)^{-1 / 4}$ and is thus compact on $L^{2}(\mathbb{R})$, see e.g. [13].

Proof of Theorem 1.1. Existence and completeness of the wave operators follows from Proposition 2.2 and Lemmas 2.3, 2.4. Absence of eigenvalues is well known, see e.g. [5].

Remark 2.5. (i) The result can be extended to the class $V=V_{1}+V_{2}$, where $V_{1}=$ $W^{\prime \prime}, W \in B^{4}(\mathbb{R})$, and $V_{2}$ satisfies $V_{2}(x)=O\left(|x|^{-1 / 2-\varepsilon}\right)$ as $x \rightarrow-\infty, V_{2}(x)=O\left(|x|^{1-\varepsilon}\right)$ as $x \rightarrow+\infty$, see $[6,14]$ for precise statements of such conditions.

(ii) The slightly complicated proof of Lemma 2.3 (ii) is needed due to a domain problem. We clearly have $\mathscr{D}(H)=\mathscr{D}\left(H_{0}\right)$, since $V$ is bounded, but in general for the class of $V$ considered here $\mathscr{D}\left(H^{2}\right) \neq \mathscr{D}\left(H_{0}^{2}\right)$. This can be seen explicitly by taking $V(x)=\sin x$.

\section{Examples of Potentials}

We give some examples of potentials satisfying our assumptions. Let $V$ be a realvalued function, which can be represented as

$$
V(x)=\int_{-\infty}^{\infty} e^{i \omega x} d \mu(\omega)
$$


where $\mu$ is a complex Borel measure which satisfies

Then it suffices to take

$$
\int_{-\infty}^{\infty}\left(\omega^{2}+\omega^{-2}\right) d|\mu|(\omega)<\infty
$$

$$
W(x)=\int_{-\infty}^{\infty}\left(-\omega^{2}\right) e^{i \omega x} d \mu(\omega)
$$

We get a large class of almost periodic functions, if we take $\mu$ to be a sum of point measures.

Another class is

$$
V=\sum_{j=1}^{N} V_{j}
$$

where $V_{j}$ is real-valued and periodic with average over a period $P_{j}$ equal zero, and $V_{j} \in C^{2}(\mathbb{R})$. This follows by taking

$$
W_{j}(x)=\int_{0}^{x} F_{j}(y) d y-c_{j} x, \quad F_{j}(x)=\int_{0}^{x} V_{j}(y) d y, \quad c_{j}=\left(\int_{0}^{P_{J}} F_{j}(y) d y\right) / P_{j} .
$$

For $V$ periodic $H=H_{0}+V$ is called a Stark-Wannier Hamiltonian. We note that the class of Hamiltonians discussed in [1] is covered by our results.

\section{A Result in Classical Particle Scattering Theory}

In this section we discuss classical particle scattering theory for Hamiltonians with the class of potentials considered above. Our result shows that the condition imposed is quite natural. Actually we can handle slightly more general potentials. We assume

$$
V(x)=U^{\prime}(x), \quad U \in C^{2,1}(\mathbb{R}) \cap B^{2}(\mathbb{R}),
$$

where $U \in C^{2,1}(\mathbb{R})$ means that $U \in C^{2}(\mathbb{R})$ and $U^{\prime \prime}$ is Lipschitz continuous.

We consider solutions to Newton's equation

$$
\ddot{x}=-1-V^{\prime}(x), \quad x(0)=x_{0}, \quad \dot{x}(0)=v_{0},
$$

where we for simplicity take the mass $m=1$. (In the quantum case we took $m=\frac{1}{2}$.)

A solution $x(t)$ to (4.2) exists globally in $t$. It is called a bound state, if

$$
\sup _{t \in \mathbb{R}}(|x(t)|+|\dot{x}(t)|)<\infty .
$$

It is called a scattering state, if there exist $\xi^{ \pm}, v^{ \pm}$, such that with

$$
y^{ \pm}(t)=-\frac{1}{2} t^{2}+t v^{ \pm}+\xi^{ \pm}
$$

we have

$$
\lim _{t \rightarrow \pm \infty}\left(\left|x(t)-y^{ \pm}(t)\right|+\left|\dot{x}(t)-\dot{y}^{ \pm}(t)\right|\right)=0,
$$

i.e. if the solution is asymptotic to a free solution for $t \rightarrow \pm \infty$. 
The main result is that asymptotic completeness holds for the class of potentials satisfying (4.1).

Theorem 4.1. Let $V$ be a real-valued function satisfying (4.1). Then there is a disjoint decomposition

$$
\mathbb{R}^{2}=M_{\text {bound }} \cup M_{\text {scat }} \cup M_{0}
$$

such that $M_{0}$ has Lebesgue measure zero, and such that $\left(x_{0}, v_{0}\right) \in$ $M_{\text {bound }}\left(\left(x_{0}, v_{0}\right) \in M_{\text {scat }}\right)$ implies that the solution $x(t)$ to (4.2) is a bound state (scattering state).

Proof. We define

$$
M_{\text {bound }}=\left\{\left(x_{0}, v_{0}\right) \mid \text { The solution } x(t) \text { to (4.2) is a bound state }\right\}
$$

and

$$
M_{\text {scat }}=\left\{\left(x_{0}, v_{0}\right) \mid \text { The solution } x(t) \text { to (4.2) is a scattering state }\right\} .
$$

We also need

$$
N_{ \pm}=\left\{\left(x_{0}, v_{0}\right) \mid \text { The solution } x(t) \text { to (4.2) satisfies } \liminf _{t \rightarrow \pm \infty} x(t)>-\infty\right\} .
$$

A well known argument due to Littlewood (see [15; XI.2]) can be applied to show that $N_{+} \backslash N_{-}$and $N_{-} \backslash N_{+}$both have Lebesgue measure zero. Let $M_{0}=$ $N_{+} \backslash N_{-} \cup N_{-} \backslash N_{+}$. It remains to show that

$$
\mathbb{R}^{2} \backslash\left(M_{0} \cup M_{\text {bound }}\right)=M_{\text {scat }} .
$$

We consider only $t \rightarrow+\infty$. The arguments for $t \rightarrow-\infty$ are entirely analogous. We assume that $x(t)$ is a solution to (4.2) which is not bound, i.e.

$$
\limsup _{t \rightarrow+\infty}(|x(t)|+|\dot{x}(t)|)=+\infty
$$

Energy is conserved, i.e.

$$
E=\frac{1}{2} \dot{x}^{2}+x+V(x)
$$

is constant. $V(x)$ bounded then implies

$$
\liminf _{t \rightarrow+\infty} x(t)=-\infty
$$

and

$$
\limsup _{t \rightarrow+\infty}|\dot{x}(t)|=+\infty
$$

It is now easy to see that there exists $t_{1}>0$ with $\dot{x}(t)<0$ for all $t \geqq t_{1}$. Therefore

$$
\lim _{t \rightarrow+\infty} x(t)=-\infty
$$

and (4.5) then implies

$$
\lim _{t \rightarrow+\infty} \dot{x}(t)=-\infty
$$


Condition (4.1) implies the existence of $c_{1}>0$ such that

$$
\left|V(x)\left(1+V^{\prime}(x)\right)\right| \leqq c_{1}
$$

for all $x \in \mathbb{R}$. We can then find $t_{0}$ such that

$$
\left|\frac{V(x(t))\left(1+V^{\prime}(x(t))\right.}{\dot{x}(t)^{2}}\right| \leqq \frac{1}{2}
$$

for all $t \geqq t_{0}$.

Integrating $\ddot{x}=-1-V^{\prime}(x)$ over $\left[t_{0}, t\right]$ we get

$$
\begin{aligned}
\dot{x}(t)-\dot{x}\left(t_{0}\right)= & -\left(t-t_{0}\right)-\int_{t_{0}}^{t} V^{\prime}(x(s)) \dot{x}(s)(\dot{x}(s))^{-1} d s \\
= & -\left(t-t_{0}\right)-V(x(t))(\dot{x}(t))^{-1}+V\left(x\left(t_{0}\right)\right)\left(\dot{x}\left(t_{0}\right)\right)^{-1} \\
& -\int_{t_{0}}^{t} V(x(s)) \ddot{x}(s)(\dot{x}(s))^{-2} d s .
\end{aligned}
$$

We chose $t_{0}$ such that the last integral is bounded by $\frac{1}{2}\left(t-t_{0}\right)$. We then get an estimate

$$
\dot{x}(t) \leqq-\frac{1}{2} t+c, \quad t \geqq t_{0},
$$

and then by iteration we see that $\dot{x}(t)+t$ has a limit as $t \rightarrow+\infty$. Explicitly we have

$$
\begin{aligned}
\dot{x}(t)+t= & t_{0}+V\left(x\left(t_{0}\right)\right)\left(\dot{x}\left(t_{0}\right)\right)^{-1}-\int_{t_{0}}^{\infty} V(x(s))\left(1+V^{\prime}(x(s))\right)(\dot{x}(s))^{-2} d s \\
& -V(x(t))(\dot{x}(t))^{-1}+\int_{t}^{\infty} V(x(s))\left(1+V^{\prime}(x(s))\right)(\dot{x}(s))^{-2} d s .
\end{aligned}
$$

Thus

$$
\lim _{t \rightarrow+\infty}(\dot{x}(t)+t)=v_{0}
$$

with $v_{0}$ given by the constant term in (4.6).

Integrating once more we find

$$
\begin{aligned}
x(t)+\frac{1}{2} t^{2}= & x\left(t_{0}\right)+\frac{1}{2} t_{0}^{2}+v_{0}\left(t-t_{0}\right)-\int_{t_{0}}^{t} V(x(s))(\dot{x}(s))^{-1} d s \\
& +\int_{t_{0}}^{t} \int_{s}^{\infty} V(x(\tau))\left(1+V^{\prime}(x(\tau))\right)(\dot{x}(\tau))^{-2} d \tau d s .
\end{aligned}
$$

The first integral in (4.7) is rewritten, using $V=U^{\prime}$

$$
\begin{aligned}
\int_{t_{0}}^{t} V(x(s)) \dot{x}(s)(\dot{x}(s))^{-2} d s= & U(x(t))(\dot{x}(t))^{-2} \\
& -U\left(x\left(t_{0}\right)\right)\left(\dot{x}\left(t_{0}\right)\right)^{-2}+2 \int_{t_{0}}^{t} U(x(s)) \ddot{x}(s)(\dot{x}(s))^{-3} d s .
\end{aligned}
$$

Thus this term tends to a constant as $t \rightarrow+\infty$. 
We rewrite the inner integral in the last term of (4.7)

$$
\begin{aligned}
\int_{s}^{\infty} V(x(\tau))\left(1+V^{\prime}(x(\tau))\right) \dot{x}(\tau)(\dot{x}(\tau))^{-3} d \tau & =\left(U(x(s))+\frac{1}{2} V(x(s))^{2}\right)(\dot{x}(s))^{-2} \\
& +3 \int_{s}^{\infty}\left(U(x(\tau))+\frac{1}{2} V(x(\tau))^{2}\right) \ddot{x}(\tau)(\dot{x}(\tau))^{-4} d \tau .
\end{aligned}
$$

Thus this integral is of order $O\left(s^{-3}\right)$ as $s \rightarrow \infty$, and therefore the double integral has a limit as $t \rightarrow+\infty$. We have then shown that

$$
\lim _{t \rightarrow+\infty}\left(x(t)+\frac{1}{2} t^{2}-t v_{0}\right)=x_{0}
$$

exists.

This proves that any state which is not bound as $t \rightarrow+\infty$ is asymptotic to a free state. With the remarks above this concludes the proof.

Remark 4.2. Classical particle scattering theory for $V$ with $V(x)=O\left(|x|^{-1 / 2-\varepsilon}\right)$ as $x \rightarrow-\infty$ has been discussed in [2].

Acknowledgements. The author wishes to thank the referee for valuable comments on the manuscript, and for suggesting looking at the classical case, and Barry Simon for his encouragement.

\section{References}

1. Agler, J., Froese, R.: Existence of Stark ladder resonances. Commun. Math. Phys. 100, 161-171 (1985)

2. Avron, J. E., Herbst, I. W.: Spectral and scattering theory for Schrödinger operators related to the Stark effect. Commun. Math. Phys. 52, 239-254 (1977)

3. Ben-Artzi, M.: Remarks on Schrödinger operators with an electric field and deterministic potentials. J. Math. Anal. Appl. 109, 333-339 (1985)

4. Bentosela, F., Carmona, R., Duclos, P., Simon, B., Souillard, B., Weder, R.: Schrödinger operators with an electric field and random or deterministic potentials. Commun. Math. Phys. 88, 387-397 (1983)

5. Dunford, N., Schwartz, J. T.: Linear operators II. New York: Wiley 1963

6. Herbst, I. W.: Unitary equivalence of Stark effect Hamiltonians. Math. Z. 155, 55-70 (1977)

7. Jensen, A., Mourre, E., Perry, P.: Multiple commutator estimates and resolvent smoothness in quantum scattering theory. Ann. Inst. H. Poincaré, Sect. A, 41, 207-225 (1984)

8. Mourre, E.: Link between the geometrical and the spectral transformation approaches in scattering theory. Commun. Math. Phys. 68, 91-94 (1979)

9. Mourre, E.: Absence of singular continuous spectrum for certain self-adjoint operators. Commun. Math. Phys. 78, 391-408 (1981)

10. Perry, P. A.: Scattering theory by the Enss method. Math. Rep. 1, part 1, 1983

11. Rejto, P. A., Sinha, K.: Absolute continuity for a 1-dimensional model of the Stark-Hamiltonian. Helv. Phys. Acta 49, 389-413 (1976)

12. Simon, B.: Phase space analysis of simple scattering systems: Extensions of some work of Enss. Duke Math. J. 46, 119-168 (1979)

13. Simon, B.: Trace ideals and their applications. Cambridge: Cambridge University Press 1977

14. Yajima, K.: Spectral and scattering theory for Schrödinger operators with Stark-effect. J. Fac. Sci. Univ. Tokyo, Sec IA, 26, 377-390 (1979)

15. Reed, M., Simon, B.: Methods of Modern Mathematical Physics. III Scattering Theory. New York: Academic Press 1979

Communicated by B. Simon

December 31, 1985; in revised form April 21, 1986 